\title{
Categorical Aspects of Intuitionistic Fuzzy Topological Spaces
}

\section{Jin Tae Kim and Seok Jong Lee}

Department of Mathematics, Chungbuk National University, Cheongju, Korea
Received: May 29, 2015

Revised : Jun. 24, 2015

Accepted: Jun. 25, 2015

Correspondence to: Seok Jong Lee (sjl@cbnu.ac.kr)

๑The Korean Institute of Intelligent Systems

cCThis is an Open Access article distributed under the terms of the Creative Commons Attribution Non-Commercial License (http://creativecommons.org/licenses/ by-nc/3.0/) which permits unrestricted noncommercial use, distribution, and reproduction in any medium, provided the original work is properly cited.

\begin{abstract}
In this paper, we obtain two types of adjoint functors between the category of intuitionistic fuzzy topological spaces in Mondal and Samanta's sense, and the category of intuitionistic fuzzy topological spaces in Šostak's sense. Also, we reveal that the category of Chang's fuzzy topological spaces is a bireflective full subcategory of the category of intuitionistic fuzzy topological spaces in Mondal and Samanta's sense.
\end{abstract}

Keywords: intuitionistic fuzzy topology

\section{Introduction}

Chang [2] defined fuzzy topological spaces with the concept of fuzzy set introduced by Zadeh [11]. After that, many generalizations of the fuzzy topology were studied by several authors like Šostak [10], Ramadan [9], and Chattopadhyay and his colleagues [3].

On the other hand, the concept of intuitionistic fuzzy sets was introduced by Atanassov [1] as a generalization of fuzzy sets. Çoker [4] introduced intuitionistic fuzzy topological spaces by using intuitionistic fuzzy sets. Mondal and Samanta [7] introduced the concept of intuitionistic gradation of openness as a generalization of a smooth topology of Ramadan (see [9]). Also, using the idea of degree of openness and degree of nonopenness, Çoker and Demirci [5] defined intuitionistic fuzzy topological spaces in Šostak's sense as a generalization of smooth topological spaces and intuitionistic fuzzy topological spaces.

Lee and Lee [6] revealed that the category of Chang's fuzzy topological spaces is a bireflective full subcategory of the category of intuitionistic fuzzy topological spaces in Çoker's sense. Also, Park and his colleagues [8] showed that the category of intuitionistic fuzzy topological spaces in Çoker's sense is a bireflective full subcategory of the category of intuitionistic fuzzy topological spaces in Šostak's sense.

The aim of this paper is to continue this investigation of categorical relationships between those categories. We obtain two types of adjoint functors between the category of intuitionistic fuzzy topological spaces in Mondal and Samanta's sense, and the category of intuitionistic fuzzy topological spaces in Šostak's sense. Also, we reveal that the category of Chang's fuzzy topological spaces is a bireflective full subcategory of the category of intuitionistic fuzzy topological spaces in Mondal and Samanta's sense.

\section{Preliminaries}

We will denote the unit interval $[0,1]$ of the real line by $I$. A member $\mu$ of $I^{X}$ is called a fuzzy 
set in $X$. By $\tilde{0}$ and $\tilde{1}$ we denote the constant fuzzy sets in $X$ with value 0 and 1 , respectively. For any $\mu \in I^{X}, \mu^{c}$ denotes the complement $\tilde{1}-\mu$.

Let $X$ be a nonempty set. An intuitionistic fuzzy set $A$ is an ordered pair

$$
A=\left(\mu_{A}, \gamma_{A}\right)
$$

where the functions $\mu_{A}: X \rightarrow I$ and $\gamma_{A}: X \rightarrow I$ denote the degree of membership and the degree of nonmembership, respectively and $\mu_{A}+\gamma_{A} \leq 1$. By $\underline{0}$ and $\underline{1}$ we denote the constant intuitionistic fuzzy sets with value $(0,1)$ and $(1,0)$, respectively. Obviously every fuzzy set $\mu$ in $X$ is an intuitionistic fuzzy set of the form $(\mu, \tilde{1}-\mu)$.

Let $f$ be a mapping from a set $X$ to a set $Y$. Let $A=$ $\left(\mu_{A}, \gamma_{A}\right)$ be an intuitionistic fuzzy set in $X$ and $B=\left(\mu_{B}, \gamma_{B}\right)$ an intuitionistic fuzzy set in $Y$. Then

(1) The image of $A$ under $f$, denoted by $f(A)$, is an intuitionistic fuzzy set in $Y$ defined by

$$
f(A)=\left(f\left(\mu_{A}\right), \tilde{1}-f\left(\tilde{1}-\gamma_{A}\right)\right) .
$$

(2) The inverse image of $B$ under $f$, denoted by $f^{-1}(B)$, is an intuitionistic fuzzy set in $X$ defined by

$$
f^{-1}(B)=\left(f^{-1}\left(\mu_{B}\right), f^{-1}\left(\gamma_{B}\right)\right) .
$$

All other notations are standard notations of fuzzy set theory.

Definition 2.1. ( [2]) A Chang's fuzzy topology on $X$ is a family $T$ of fuzzy sets in $X$ which satisfies the following properties:

(1) $\tilde{0}, \tilde{1} \in T$.

(2) If $\mu_{1}, \mu_{2} \in T$, then $\mu_{1} \wedge \mu_{2} \in T$.

(3) If $\mu_{i} \in T$ for each $i$, then $\bigvee \mu_{i} \in T$.

The pair $(X, T)$ is called a fuzzy topological space.

Definition 2.2. ( [9]) A smooth topology on $X$ is a mapping $T: I^{X} \rightarrow I$ which satisfies the following properties:

(1) $T(\tilde{0})=T(\tilde{1})=1$.

(2) $T\left(\mu_{1} \wedge \mu_{2}\right) \geq T\left(\mu_{1}\right) \wedge T\left(\mu_{2}\right)$.

(3) $T\left(\bigvee \mu_{i}\right) \geq \bigwedge T\left(\mu_{i}\right)$.

The pair $(X, T)$ is called a smooth topological space.
Definition 2.3. ( [4]) An intuitionistic fuzzy topology on $X$ is a family $T$ of intuitionistic fuzzy sets in $X$ which satisfies the following properties:

(1) $\underline{0}, \underline{1} \in T$.

(2) If $A_{1}, A_{2} \in T$, then $A_{1} \cap A_{2} \in T$.

(3) If $A_{i} \in T$ for each $i$, then $\bigcup A_{i} \in T$.

The pair $(X, T)$ is called an intuitionistic fuzzy topological space.

Let $I(X)$ be a family of all intuitionistic fuzzy sets in $X$ and let $I \otimes I$ be the set of the pair $(r, s)$ such that $r, s \in I$ and $r+s \leq 1$.

Definition 2.4. ( [5]) Let $X$ be a nonempty set. An intuitionistic fuzzy topology in Šostak's sense(SoIFT for short) $\mathcal{T}=\left(\mathcal{T}_{1}, \mathcal{T}_{2}\right)$ on $X$ is a mapping $\mathcal{T}: I(X) \rightarrow I \otimes I$ which satisfies the following properties:

(1) $\mathcal{T}_{1}(\underline{0})=\mathcal{T}_{1}(\underline{1})=1$ and $\mathcal{T}_{2}(\underline{0})=\mathcal{T}_{2}(\underline{1})=0$.

(2) $\mathcal{T}_{1}(A \cap B) \geq \mathcal{T}_{1}(A) \wedge \mathcal{T}_{1}(B)$ and $\mathcal{T}_{2}(A \cap B) \leq \mathcal{T}_{2}(A) \vee$ $\mathcal{T}_{2}(B)$.

(3) $\mathcal{T}_{1}\left(\bigcup A_{i}\right) \geq \bigwedge \mathcal{T}_{1}\left(A_{i}\right)$ and $\mathcal{T}_{2}\left(\bigcup A_{i}\right) \leq \bigvee \mathcal{T}_{2}\left(A_{i}\right)$.

Then $(X, \mathcal{T})=\left(X, \mathcal{T}_{1}, \mathcal{T}_{2}\right)$ is said to be an intuitionistic fuzzy topological space in Šostak's sense(SoIFTS for short). Also, we call $\mathcal{T}_{1}(A)$ the gradation of openness of $A$ and $\mathcal{T}_{2}(A)$ the gradation of nonopenness of $A$.

Definition 2.5. ( [5]) Let $f:\left(X, \mathcal{T}_{1}, \mathcal{T}_{2}\right) \rightarrow\left(Y, \mathcal{U}_{1}, \mathcal{U}_{2}\right)$ be a mapping from a SoIFTS $X$ to a SoIFTS $Y$. Then $f$ is said to be SoIF continuous if $\mathcal{T}_{1}\left(f^{-1}(B)\right) \geq \mathcal{T}_{1}(B)$ and $\mathcal{T}_{2}\left(f^{-1}(B)\right) \leq$ $\mathcal{T}_{2}(B)$ for each $B \in I(Y)$

Let $(X, \mathcal{T})$ be a SoIFTS. Then for each $(r, s) \in I \otimes I$, the family $\mathcal{T}_{(r, s)}$ defined by

$$
\mathcal{T}_{(r, s)}=\left\{A \in I(X) \mid \mathcal{T}_{1}(A) \geq r \text { and } \mathcal{T}_{2}(A) \leq s\right\}
$$

is an intuitionistic fuzzy topology on $X$. In this case, $\mathcal{T}_{(r, s)}$ is called the $(r, s)$-level intuitionistic fuzzy topology on $X$.

Let $(X, T)$ be an intuitionistic fuzzy topological space. Then for each $(r, s) \in I \otimes I$, a SoIFT $T^{(r, s)}: I(X) \rightarrow I \otimes I$ defined by

$$
T^{(r, s)}(A)= \begin{cases}(1,0) & \text { if } A=\underline{0}, \underline{1}, \\ (r, s) & \text { if } A \in T-\{\underline{0}, \underline{1}\} \\ (0,1) & \text { otherwise. }\end{cases}
$$


In this case, $T^{(r, s)}$ is called an $(r, s)$-th graded SoIFT on $X$ and $\left(X, T^{(r, s)}\right)$ is called an $(r, s)$-th graded SoIFTS on $X$.

Definition 2.6. ( [7]) Let $X$ be a nonempty set. An intuitionistic fuzzy topology in Mondal and Samanta's sense(MSIFT for short) $T=\left(T_{1}, T_{2}\right)$ on $X$ is a mapping $T: I^{X} \rightarrow I \otimes I$ which satisfy the following properties:

(1) $T_{1}(\tilde{0})=T_{1}(\tilde{1})=1$ and $T_{2}(\tilde{0})=T_{2}(\tilde{1})=0$.

(2) $T_{1}(\mu \wedge \eta) \geq T_{1}(\mu) \wedge T_{1}(\eta)$ and $T_{2}(\mu \wedge \eta) \leq T_{2}(\mu) \vee$ $T_{2}(\eta)$

(3) $T_{1}\left(\bigvee \mu_{i}\right) \geq \bigwedge T_{1}\left(\mu_{i}\right)$ and $T_{2}\left(\bigvee \mu_{i}\right) \leq \bigvee T_{2}\left(\mu_{i}\right)$.

Then $(X, T)$ is said to be an intuitionistic fuzzy topological space in Mondal and Samanta's sense(MSIFTS for short). $T_{1}$ and $T_{2}$ may be interpreted as gradation of openness and gradation of nonopenness, respectively.

Definition 2.7. ( [7]) Let $f:\left(X, T_{1}, T_{2}\right) \rightarrow\left(Y, U_{1}, U_{2}\right)$ be a mapping. Then $f$ is said to be MSIF contiunous if $T_{1}\left(f^{-1}(\eta)\right) \geq$ $U_{1}(\eta)$ and $T_{2}\left(f^{-1}(\eta)\right) \leq U_{2}(\eta)$ for each $\eta \in I^{Y}$.

Let $(X, T)$ be a MSIFTS. Then for each $(r, s) \in I \otimes I$, the family $T_{(r, s)}$ defined by

$$
T_{(r, s)}=\left\{\mu \in I^{X} \mid T_{1}(\mu) \geq r \text { and } T_{2}(\mu) \leq s\right\}
$$

is a Chang's fuzzy topology on $X$. In this case, $T_{(r, s)}$ is called the $(r, s)$-level Chang's fuzzy topology on $X$.

Let $(X, T)$ be a Chang's fuzzy topological spaces. Then for each $(r, s) \in I \otimes I$, a MSIFT $T^{(r, s)}: I^{X} \rightarrow I \otimes I$ is defined by

$$
T^{(r, s)}(\mu)= \begin{cases}(1,0) & \text { if } \mu=\tilde{0}, \tilde{1} \\ (r, s) & \text { if } \mu \in T-\{\tilde{0}, \tilde{1}\} \\ (0,1) & \text { otherwise. }\end{cases}
$$

In this case, $T^{(r, s)}$ is called an $(r, s)$-th graded MSIFT on $X$ and $\left(X, T^{(r, s)}\right)$ is called an $(r, s)$-th graded MSIFTS on $X$.

\section{The categorical relationships between MSIFTop and SoIFTop}

Let MSIFTop be the category of all intuitionistic fuzzy topological spaces in Mondal and Samanta's sense and MSIF continuous mappings, and let SoIFTop be the category of all intuitionistic fuzzy topological spaces in Šostak's sense and SoIF continuous mappings.
Theorem 3.1. Define a functor $F:$ SoIFTop $\rightarrow$ MSIFTop by $F(X, \mathcal{T})=(X, F(\mathcal{T}))$ and $F(f)=f$, where $F(\mathcal{T})(\eta)=$ $\left(F(\mathcal{T})_{1}(\eta), F(\mathcal{T})_{2}(\eta)\right), F(\mathcal{T})_{1}(\eta)=\bigvee\left\{\mathcal{T}_{1}(A) \mid \mu_{A}=\eta\right\}$ $F(\mathcal{T})_{2}(\eta)=\bigwedge\left\{\mathcal{T}_{2}(A) \mid \mu_{A}=\eta\right\}$. Then $F$ is a functor.

Proof. First, we show that $F(\mathcal{T})$ is a MSIFT.

Clearly, $F(\mathcal{T})(\eta)=F(\mathcal{T})_{1}(\eta)+F(\mathcal{T})_{2}(\eta) \leq 1$ for any $\eta \in$ $I^{X}$.

(1) $F(\mathcal{T})_{1}(\tilde{0})=\bigvee\left\{\mathcal{T}_{1}(A) \mid \mu_{A}=\tilde{0}\right\} \geq \mathcal{T}_{1}(\underline{0})=1$, $F(\mathcal{T})_{1}(\tilde{1})=\bigvee\left\{\mathcal{T}_{1}(A) \mid \mu_{A}=\tilde{1}\right\} \geq \mathcal{T}_{1}(\underline{1})=1, F(\mathcal{T})_{2}(\tilde{0})=$ $\bigwedge\left\{\mathcal{T}_{2}(A) \mid \mu_{A}=\tilde{0}\right\} \leq \mathcal{T}_{2}(\underline{0})=0$, and $F(\mathcal{T})_{2}(\tilde{1})=\bigwedge\left\{\mathcal{T}_{2}(A) \mid\right.$ $\left.\mu_{A}=\tilde{1}\right\} \leq \mathcal{T}_{2}(\underline{1})=0$.

(2) Suppose that $F(\mathcal{T})_{1}(\eta \wedge \lambda)<F(\mathcal{T})_{1}(\eta) \wedge F(\mathcal{T})_{1}(\lambda)$. Then there is a $t \in I$ such that $F(\mathcal{T})_{1}(\eta \wedge \lambda)<t<F(\mathcal{T})_{1}(\eta) \wedge$ $F(\mathcal{T})_{1}(\lambda)$. Since $t<F(\mathcal{T})_{1}(\eta)=\bigvee\left\{\mathcal{T}_{1}(C) \mid \mu_{C}=\eta\right\}$, there is an $A \in I(X)$ such that $t<\mathcal{T}_{1}(A)$ and $\mu_{A}=\eta$. There is a $B \in I(X)$ such that $t<\mathcal{T}_{1}(B)$ and $\mu_{B}=\lambda$, because $t<F(\mathcal{T})_{1}(\lambda)=\bigvee\left\{\mathcal{T}_{1}(C) \mid \mu_{C}=\lambda\right\}$. Thus $t<\mathcal{T}_{1}(A) \wedge \mathcal{T}_{1}(B)$ and $\mu_{A \cap B}=\mu_{A} \wedge \mu_{B}=\eta \wedge \lambda$. Since $\mathcal{T}$ is a SoIFT, we obtain

$$
t<\mathcal{T}_{1}(A) \wedge \mathcal{T}_{1}(B) \leq \mathcal{T}_{1}(A \cap B)
$$

Hence

$$
\begin{aligned}
t & >F(\mathcal{T})_{1}(\eta \wedge \lambda)=\bigvee\left\{\mathcal{T}_{1}(C) \mid \mu_{C}=\eta \wedge \lambda\right\} \\
& \geq \mathcal{T}_{1}(A \cap B) \geq \mathcal{T}_{1}(A) \wedge \mathcal{T}_{1}(B)>t
\end{aligned}
$$

This is a contradiction. Thus $F(\mathcal{T})_{1}(\eta \wedge \lambda) \geq F(\mathcal{T})_{1}(\eta) \wedge$ $F(\mathcal{T})_{2}(\lambda)$.

Next, assume that $F(\mathcal{T})_{2}(\eta \wedge \lambda)>F(\mathcal{T})_{2}(\eta) \vee F(\mathcal{T})_{2}(\lambda)$. Then there is an $s \in I$ such that

$$
F(\mathcal{T})_{2}(\eta \wedge \lambda)>s>F(\mathcal{T})_{2}(\eta) \vee F(\mathcal{T})_{2}(\lambda)
$$

Since $s>F(\mathcal{T})_{2}(\eta)=\bigwedge\left\{\mathcal{T}_{2}(C) \mid \mu_{C}=\eta\right\}$, there is an $A \in$ $I(X)$ such that $s>\mathcal{T}_{2}(A)$ and $\mu_{A}=\eta$. As $s>F(\mathcal{T})_{2}(\lambda)=$ $\bigwedge\left\{\mathcal{T}_{2}(C) \mid \mu_{C}=\lambda\right\}$, there is a $B \in I(X)$ such that $s>$ $\mathcal{T}_{2}(B)$ and $\mu_{B}=\lambda$. So $s>\mathcal{T}_{2}(A) \vee \mathcal{T}_{2}(B)$ and $\mu_{A \cap B}=$ $\mu_{A} \wedge \mu_{B}=\eta \wedge \lambda$. Since $\mathcal{T}$ is a SoIFT, we have $s>\mathcal{T}_{2}(A) \vee$ $\mathcal{T}_{2}(B) \geq \mathcal{T}_{2}(A \cap B)$. Thus

$$
\begin{aligned}
s & <F(\mathcal{T})_{2}(\eta \wedge \lambda)=\bigwedge\left\{\mathcal{T}_{2}(C) \mid \mu_{C}=\eta \wedge \lambda\right\} \\
& \leq \mathcal{T}_{2}(A \cap B) \leq \mathcal{T}_{2}(A) \vee \mathcal{T}_{2}(B)<s .
\end{aligned}
$$

This is a contradiction. Hence $F(\mathcal{T})_{2}(\eta \wedge \lambda) \leq F(\mathcal{T})_{2}(\eta) \vee$ $F(\mathcal{T})_{2}(\lambda)$. 
(3) Suppose that $F(\mathcal{T})_{1}\left(\bigvee \eta_{i}\right)<\bigwedge F(\mathcal{T})_{1}\left(\eta_{i}\right)$. Then there is a $t \in I$ such that $F(\mathcal{T})_{1}\left(\bigvee \eta_{i}\right)<t<\bigwedge F(\mathcal{T})_{1}\left(\eta_{i}\right)$. Since $t<F(\mathcal{T})_{1}\left(\eta_{i}\right)=\bigvee\left\{\mathcal{T}_{1}(C) \mid \mu_{C}=\eta_{i}\right\}$ for each $i$, there is an $A_{i} \in I(X)$ such that $t<\mathcal{T}_{1}\left(A_{i}\right)$ and $\mu_{A_{i}}=\eta_{i}$. Thus $t \leq \bigwedge \mathcal{T}_{1}\left(A_{i}\right)$ and $\mu_{\cup A_{i}}=\bigvee \mu_{A_{i}}=\bigvee \eta_{i}$. As $\mathcal{T}$ is a SoIFT, we obtain $\mathcal{T}_{1}\left(\bigcup A_{i}\right) \geq \bigwedge \mathcal{T}_{1}\left(A_{i}\right)$. Hence

$$
\begin{aligned}
t & >F(\mathcal{T})_{1}\left(\bigvee \eta_{i}\right)=\bigvee\left\{\mathcal{T}_{1}(C) \mid \mu_{C}=\bigvee \eta_{i}\right\} \\
& \geq \mathcal{T}_{1}\left(\bigcup A_{i}\right) \geq \bigwedge \mathcal{T}_{1}\left(A_{i}\right) \geq t .
\end{aligned}
$$

This is a contradiction. Thus $F(\mathcal{T})_{1}\left(\bigvee \eta_{i}\right) \geq \bigwedge F(\mathcal{T})_{1}\left(\eta_{i}\right)$.

Next, assume that $F(\mathcal{T})_{2}\left(\bigvee \eta_{i}\right)>\bigvee F(\mathcal{T})_{2}\left(\eta_{i}\right)$. Then there is an $s \in I$ such that

$$
F(\mathcal{T})_{2}\left(\bigvee \eta_{i}\right)>s>\bigvee F(\mathcal{T})_{2}\left(\eta_{i}\right)
$$

Since $s>F(\mathcal{T})_{2}\left(\eta_{i}\right)=\bigwedge\left\{\mathcal{T}_{2}(C) \mid \mu_{C}=\eta_{i}\right\}$ for each $i$, there is a $B_{i} \in I(X)$ such that $s>\mathcal{T}_{2}\left(B_{i}\right)$ and $\mu_{B_{i}}=\eta_{i}$. Hence $s \geq \bigvee \mathcal{T}_{2}\left(B_{i}\right)$ and $\mu_{\cup B_{i}}=\bigvee \mu_{B_{i}}=\bigvee \eta_{i}$. Since $\mathcal{T}$ is a SoIFT, we have $\mathcal{T}_{2}\left(\bigcup B_{i}\right) \leq \bigvee \mathcal{T}_{2}\left(B_{i}\right)$. Thus

$$
\begin{aligned}
s & <F(\mathcal{T})_{2}\left(\bigvee \eta_{i}\right)=\bigwedge\left\{\mathcal{T}_{2}(C) \mid \mu_{C}=\bigvee \eta_{i}\right\} \\
& \leq \mathcal{T}_{2}\left(\bigcup B_{i}\right) \leq \bigvee \mathcal{T}_{2}\left(B_{i}\right) \leq s .
\end{aligned}
$$

This is a contradiction. Hence $F(\mathcal{T})_{2}\left(\bigvee \eta_{i}\right) \leq \bigvee F(\mathcal{T})_{2}\left(\eta_{i}\right)$ Therefore $(X, F(\mathcal{T}))$ is a MSIFTS.

Finally, we show that if $f:(X, \mathcal{T}) \rightarrow(Y, \mathcal{U})$ is SoIF continuous, then $f:(X, F(\mathcal{T})) \rightarrow(Y, F(\mathcal{U}))$ is MSIF continuous. Let $F(\mathcal{T})=\left(F(\mathcal{T})_{1}, F(\mathcal{T})_{2}\right), F(\mathcal{U})=\left(F(\mathcal{U})_{1}, F(\mathcal{U})_{2}\right)$, and $\lambda \in I^{Y}$. Then

$$
\begin{aligned}
& F(\mathcal{U})_{1}(\lambda)=\bigvee\left\{\mathcal{U}_{1}(A) \mid \mu_{A}=\lambda\right\} \\
\leq & \bigvee\left\{\mathcal{T}_{1}\left(f^{-1}(A)\right) \mid \mu_{f^{-1}(A)}=f^{-1}(\lambda)\right\} \\
\leq & \bigvee\left\{\mathcal{T}_{1}(C) \mid \mu_{C}=f^{-1}(\lambda)\right\}=F(\mathcal{T})_{1}\left(f^{-1}(\lambda)\right)
\end{aligned}
$$

and

$$
\begin{aligned}
& F(\mathcal{U})_{2}(\lambda)=\bigwedge\left\{\mathcal{U}_{2}(A) \mid \mu_{A}=\lambda\right\} \\
\geq & \bigwedge\left\{\mathcal{T}_{2}\left(f^{-1}(A)\right) \mid \mu_{f^{-1}(A)}=f^{-1}(\lambda)\right\} \\
\geq & \bigwedge\left\{\mathcal{T}_{2}(C) \mid \mu_{C}=f^{-1}(\lambda)\right\}=F(\mathcal{T})_{2}\left(f^{-1}(\lambda)\right)
\end{aligned}
$$

Therefore $F$ is a functor.

Theorem 3.2. Define a functor $G:$ MSIFTop $\rightarrow$ SoIFTop by $G(X, T)=(X, G(T))$ and $G(f)=f$, where $G(T)(A)=$
$\left(G(T)_{1}(A), G(T)_{2}(A)\right), G(T)_{1}(A)=T_{1}\left(\mu_{A}\right)$, and $G(T)_{2}(A)=T_{2}\left(\mu_{A}\right)$. Then $G$ is a functor.

Proof. First, we show that $G(T)$ is a SoIFT.

Clearly, $G(T)_{1}(A)+G(T)_{2}(A)=T_{1}\left(\mu_{A}\right)+T_{2}\left(\mu_{A}\right) \leq 1$ for any $A \in I(X)$.

(1) $G(T)_{1}(\underline{0})=T_{1}(\tilde{0})=1, G(T)_{1}(\underline{1})=T_{1}(\tilde{1})=1$, $G(T)_{2}(\underline{0})=T_{2}(\tilde{0})=0$, and $G(T)_{2}(\underline{1})=T_{2}(\tilde{1})=0$.

(2) Let $A, B \in I(X)$. Then

$$
\begin{aligned}
G(T)_{1}(A \cap B) & =T_{1}\left(\mu_{A \cap B}\right)=T_{1}\left(\mu_{A} \wedge \mu_{B}\right) \\
& \geq T_{1}\left(\mu_{A}\right) \wedge T_{1}\left(\mu_{B}\right) \\
& =G(T)_{1}(A) \wedge G(T)_{1}(B)
\end{aligned}
$$

and

$$
\begin{aligned}
G(T)_{2}(A \cap B) & =T_{2}\left(\mu_{A \cap B}\right)=T_{2}\left(\mu_{A} \wedge \mu_{B}\right) \\
& \leq T_{2}\left(\mu_{A}\right) \vee T_{2}\left(\mu_{B}\right) \\
& =G(T)_{2}(A) \vee G(T)_{2}(B) .
\end{aligned}
$$

(3) Let $A_{i} \in I(X)$ for each $i$. Then

$$
\begin{aligned}
G(T)_{1}\left(\bigcup A_{i}\right) & =T_{1}\left(\mu_{\cup A_{i}}\right)=T_{1}\left(\bigvee \mu_{A_{i}}\right) \\
& \geq \bigwedge T_{1}\left(\mu_{A_{i}}\right)=\bigwedge G(T)_{1}\left(A_{i}\right)
\end{aligned}
$$

and

$$
\begin{aligned}
G(T)_{2}\left(\bigcup A_{i}\right) & =T_{2}\left(\mu_{\cup A_{i}}\right)=T_{2}\left(\bigvee \mu_{A_{i}}\right) \\
& \leq \bigvee T_{2}\left(\mu_{A_{i}}\right)=\bigvee G(T)_{2}\left(A_{i}\right)
\end{aligned}
$$

Hence $(X, G(T))$ is a SoIFT.

Next, we show that if $f:(X, T) \rightarrow(Y, U)$ is MSIF continuous, then $f:(X, G(T)) \rightarrow(Y, G(U))$ is SoIF continuous. Let $B=\left(\mu_{B}, \gamma_{B}\right) \in I(Y)$. Then

$$
\begin{aligned}
G(U)_{1}(B) & =U_{1}\left(\mu_{B}\right) \leq T_{1}\left(f^{-1}\left(\mu_{B}\right)\right)=T_{1}\left(\mu_{f^{-1}(B)}\right) \\
& =G(T)_{1}\left(f^{-1}(B)\right)
\end{aligned}
$$

and

$$
\begin{aligned}
G(U)_{2}(B) & =U_{2}\left(\mu_{B}\right) \geq T_{2}\left(f^{-1}\left(\mu_{B}\right)\right)=T_{2}\left(\mu_{f^{-1}(B)}\right) \\
& =G(T)_{2}\left(f^{-1}(B)\right) .
\end{aligned}
$$

Thus $f:(X, G(T)) \rightarrow(Y, G(U))$ is SoIF continuous. Consequently, $G$ is a functor. 
Theorem 3.3. The functor $G:$ MSIFTop $\rightarrow$ SoIFTop is a left adjoint of $F$ : SoIFTop $\rightarrow$ MSIFTop.

Proof. Let $(X, T)$ be an object in MSIFTop and $\eta \in I^{X}$.

Then

$$
\begin{aligned}
& F G(T)(\eta) \\
= & \left(\bigvee\left\{G(T)_{1}(A) \mid \mu_{A}=\eta\right\}, \bigwedge\left\{G(T)_{2}(A) \mid \mu_{A}=\eta\right\}\right) \\
= & \left(\bigvee\left\{T_{1}\left(\mu_{A}\right) \mid \mu_{A}=\eta\right\}, \bigwedge\left\{T_{2}\left(\mu_{A}\right) \mid \mu_{A}=\eta\right\}\right) \\
= & \left(T_{1}(\eta), T_{2}(\eta)\right)=T(\eta) .
\end{aligned}
$$

Hence $1_{X}:(X, T) \rightarrow F G(X, T)=(X, T)$ is MSIF continuous.

Consider $(Y, \mathcal{U}) \in$ SoIFTop and a MSIF continuous mapping $f:(X, T) \rightarrow F(Y, \mathcal{U})$. In order to show that $f:$ $G(X, T) \rightarrow(Y, \mathcal{U})$ is a SoIF continuous mapping, let $B \in$ $I(Y)$. Then

$$
\begin{aligned}
G(T)_{1}\left(f^{-1}(B)\right) & =T_{1}\left(\mu_{f^{-1}(B)}\right)=T_{1}\left(f^{-1}\left(\mu_{B}\right)\right) \\
& \left.\geq F(\mathcal{U})_{1}\left(\mu_{B}\right)\right)=\bigvee\left\{\mathcal{U}_{1}(C) \mid \mu_{C}=\mu_{B}\right\} \\
& \geq \mathcal{U}_{1}(B)
\end{aligned}
$$

and

$$
\begin{aligned}
G(T)_{2}\left(f^{-1}(B)\right) & =T_{2}\left(\mu_{f^{-1}(B)}\right)=T_{2}\left(f^{-1}\left(\mu_{B}\right)\right) \\
& \left.\leq F(\mathcal{U})_{2}\left(\mu_{B}\right)\right)=\bigwedge\left\{\mathcal{U}_{2}(C) \mid \mu_{C}=\mu_{B}\right\} \\
& \leq \mathcal{U}_{2}(B) .
\end{aligned}
$$

Hence $f:\left(X, G(T)_{1}, G(T)_{2}\right) \rightarrow\left(Y, \mathcal{U}_{1}, \mathcal{U}_{2}\right)$ is a SoIF continuous mapping. Therefore $1_{X}$ is a $G$-universal mapping for $(X, T)$ in MSIFTop.

Theorem 3.4. Define a functor $H$ : SoIFTop $\rightarrow$ MSIFTop by $H(X, \mathcal{T})=(X, H(\mathcal{T}))$ and $H(f)=f$, where $H(\mathcal{T})=$ $\left(H(\mathcal{T})_{1}, H(\mathcal{T})_{2}\right), H(\mathcal{T})_{1}(\eta)=\bigvee\left\{\mathcal{T}_{1}(A) \mid \tilde{1}-\gamma_{A}=\eta\right\}$, and $H(\mathcal{T})_{2}(\eta)=\bigwedge\left\{\mathcal{T}_{2}(A) \mid \tilde{1}-\gamma_{A}=\eta\right\}$. Then $H$ is a functor.

Proof. First, we show that $H(\mathcal{T})$ is a MSIFT. Obviously, $H(\mathcal{T})(\eta)=H(\mathcal{T})_{1}(\eta)+H(\mathcal{T})_{2}(\eta) \leq 1$ for any $\eta \in I^{X}$

(1) $H(\mathcal{T})_{1}(\tilde{0})=\bigvee\left\{\mathcal{T}_{1}(A) \mid \tilde{1}-\gamma_{A}=\tilde{0}\right\} \geq \mathcal{T}_{1}(\underline{0})=$ $1, H(\mathcal{T})_{1}(\tilde{1})=\bigvee\left\{\mathcal{T}_{1}(A) \mid \tilde{1}-\gamma_{A}=\tilde{1}\right\} \geq \mathcal{T}_{1}(\underline{1})=1$, $H(\mathcal{T})_{2}(\tilde{0})=\bigwedge\left\{\mathcal{T}_{2}(A) \mid \tilde{1}-\gamma_{A}=\tilde{0}\right\} \leq \mathcal{T}_{2}(\underline{0})=0$, and $H(\mathcal{T})_{2}(\tilde{1})=\bigwedge\left\{\mathcal{T}_{2}(A) \mid \tilde{1}-\gamma_{A}=\tilde{1}\right\} \leq \mathcal{T}_{2}(\underline{1})=0$.

(2) Assume that $H(\mathcal{T})_{1}(\eta \wedge \lambda)<H(\mathcal{T})_{1}(\eta) \wedge H(\mathcal{T})_{1}(\lambda)$.
Then there is a $t \in I$ such that

$$
H(\mathcal{T})_{1}(\eta \wedge \lambda)<t<H(\mathcal{T})_{1}(\eta) \wedge H(\mathcal{T})_{1}(\lambda) .
$$

As $t<H(\mathcal{T})_{1}(\eta)=\bigvee\left\{\mathcal{T}_{1}(C) \mid \tilde{1}-\gamma_{C}=\eta\right\}$, there is an $A \in I(X)$ such that $t<\mathcal{T}_{1}(A)$ and $\tilde{1}-\gamma_{A}=\eta$. Since $t<$ $H(\mathcal{T})_{1}(\lambda)=\bigvee\left\{\mathcal{T}_{1}(C) \mid \tilde{1}-\gamma_{C}=\lambda\right\}$, there is a $B \in I(X)$ such that $t<\mathcal{T}_{1}(B)$ and $\tilde{1}-\gamma_{B}=\lambda$. Hence $t<\mathcal{T}_{1}(A) \wedge \mathcal{T}_{1}(B)$ and

$$
\begin{aligned}
\tilde{1}-\gamma_{A \cap B} & =\tilde{1}-\left(\gamma_{A} \vee \gamma_{B}\right) \\
& =\left(\tilde{1}-\gamma_{A}\right) \wedge\left(\tilde{1}-\gamma_{B}\right)=\eta \wedge \lambda .
\end{aligned}
$$

Since $\mathcal{T}$ is a SoIFT, $t<\mathcal{T}_{1}(A) \wedge \mathcal{T}_{1}(B) \leq \mathcal{T}_{1}(A \cap B)$. Thus

$$
\begin{aligned}
t & >H(\mathcal{T})_{1}(\eta \wedge \lambda)=\bigvee\left\{\mathcal{T}_{1}(C) \mid \tilde{1}-\gamma_{C}=\eta \wedge \lambda\right\} \\
& \geq \mathcal{T}_{1}(A \cap B) \geq \mathcal{T}_{1}(A) \wedge \mathcal{T}_{1}(B)>t
\end{aligned}
$$

This is a contradiction. Hence $H(\mathcal{T})_{1}(\eta \wedge \lambda) \geq H(\mathcal{T})_{1}(\eta) \wedge$ $H(\mathcal{T})_{1}(\lambda)$.

Suppose that $H(\mathcal{T})_{2}(\eta \wedge \lambda)>H(\mathcal{T})_{2}(\eta) \vee H(\mathcal{T})_{2}(\lambda)$. Then there is an $s \in I$ such that

$$
H(\mathcal{T})_{2}(\eta \wedge \lambda)>s>H(\mathcal{T})_{2}(\eta) \vee H(\mathcal{T})_{2}(\lambda)
$$

Since $s>H(\mathcal{T})_{2}(\eta)=\bigwedge\left\{\mathcal{T}_{2}(C) \mid \tilde{1}-\gamma_{C}=\eta\right\}$, there is an $A \in I(X)$ such that $s>\mathcal{T}_{2}(A)$ and $\tilde{1}-\gamma_{A}=\eta$. As $s>$ $H(\mathcal{T})_{2}(\lambda)=\bigwedge\left\{\mathcal{T}_{2}(C) \mid \tilde{1}-\gamma_{C}=\lambda\right\}$, there is a $B \in I(X)$ such that $s>\mathcal{T}_{2}(B)$ and $\tilde{1}-\gamma_{B}=\lambda$. So $s>\mathcal{T}_{2}(A) \vee \mathcal{T}_{2}(B)$ and

$$
\begin{aligned}
\tilde{1}-\gamma_{A \cap B} & =\tilde{1}-\left(\gamma_{A} \vee \gamma B\right) \\
& =\left(\tilde{1}-\gamma_{A}\right) \wedge\left(\tilde{1}-\gamma_{B}\right)=\eta \wedge \lambda .
\end{aligned}
$$

Since $\mathcal{T}$ is a SoIFT, we obtain $s>\mathcal{T}_{2}(A) \vee \mathcal{T}_{2}(B) \geq \mathcal{T}_{2}(A \cap B)$. Hence

$$
\begin{aligned}
s & <H(\mathcal{T})_{2}(\eta \wedge \lambda)=\bigwedge\left\{\mathcal{T}_{2}(C) \mid \tilde{1}-\gamma_{C}=\eta \wedge \lambda\right\} \\
& \leq \mathcal{T}_{2}(A \cap B) \leq \mathcal{T}_{2}(A) \vee \mathcal{T}_{2}(B)<s .
\end{aligned}
$$

This is a contradiction. Thus $H(\mathcal{T})_{2}(\eta \wedge \lambda) \leq H(\mathcal{T})_{2}(\eta) \vee$ $H(\mathcal{T})_{2}(\lambda)$

(3) Assume that $H(\mathcal{T})_{1}\left(\bigvee \eta_{i}\right)<\bigwedge H(\mathcal{T})_{1}\left(\eta_{i}\right)$. Then there is a $t \in I$ such that

$$
H(\mathcal{T})_{1}\left(\bigvee \eta_{i}\right)<t<\bigwedge H(\mathcal{T})_{1}\left(\eta_{i}\right)
$$


As $t<H(\mathcal{T})_{1}\left(\eta_{i}\right)=\bigvee\left\{\mathcal{T}_{1}(C) \mid \tilde{1}-\gamma_{C}=\eta_{i}\right\}$ for each $i$, there is an $A_{i} \in I(X)$ such that $t<\mathcal{T}_{1}\left(A_{i}\right)$ and $\tilde{1}-\gamma_{A_{i}}=\eta_{i}$. Hence $t \leq \bigwedge \mathcal{T}_{1}\left(A_{i}\right)$ and

$$
\tilde{1}-\gamma_{\left(\cup A_{i}\right)}=\tilde{1}-\bigwedge \gamma_{A_{i}}=\bigvee\left(\tilde{1}-\gamma_{A_{i}}\right)=\bigvee \eta_{i} .
$$

Since $\mathcal{T}$ is a SoIFT, we have $\mathcal{T}_{1}\left(\bigcup A_{i}\right) \geq \bigwedge \mathcal{T}_{1}\left(A_{i}\right)$. Thus

$$
\begin{aligned}
t & >H(\mathcal{T})_{1}\left(\bigvee \eta_{i}\right)=\bigvee\left\{\mathcal{T}_{1}(C) \mid \tilde{1}-\gamma_{C}=\bigvee \eta_{i}\right\} \\
& \geq \mathcal{T}_{1}\left(\bigcup A_{i}\right) \geq \bigwedge \mathcal{T}_{1}\left(A_{i}\right) \geq t
\end{aligned}
$$

This is a contradiction. Hence $H(\mathcal{T})_{1}\left(\bigvee \eta_{i}\right) \geq \bigwedge H(\mathcal{T})_{1}\left(\eta_{i}\right)$.

Suppose that $H(\mathcal{T})_{2}\left(\bigvee \eta_{i}\right)>\bigvee H(\mathcal{T})_{2}\left(\eta_{i}\right)$. Then there is an $s \in I$ such that $H(\mathcal{T})_{2}\left(\bigvee \eta_{i}\right)>s>\bigvee H(\mathcal{T})_{2}\left(\eta_{i}\right)$. Since $s>H(\mathcal{T})_{2}\left(\eta_{i}\right)=\bigwedge\left\{\mathcal{T}_{2}(C) \mid \tilde{1}-\gamma_{C}=\eta_{i}\right\}$ for each $i$, there is a $B_{i} \in I(X)$ such that $s>\mathcal{T}_{2}\left(B_{i}\right)$ and $\tilde{1}-\gamma_{B_{i}}=\eta_{i}$. Hence $s \geq \bigvee \mathcal{T}_{2}\left(B_{i}\right)$ and

$$
\tilde{1}-\gamma_{B_{i}}=\tilde{1}-\bigwedge \gamma_{B_{i}}=\bigvee\left(\tilde{1}-\gamma_{B_{i}}\right)=\bigvee \eta_{i} .
$$

We have $\mathcal{T}_{2}\left(\bigcup B_{i}\right) \leq \bigvee \mathcal{T}_{2}\left(B_{i}\right)$ because $\mathcal{T}$ is a SoIFT. Thus

$$
\begin{aligned}
s & <H(\mathcal{T})_{2}\left(\bigvee \eta_{i}\right)=\bigwedge\left\{\mathcal{T}_{2}(C) \mid \tilde{1}-\gamma_{C}=\bigvee \eta_{i}\right\} \\
& \leq \mathcal{T}_{2}\left(\bigcup B_{i}\right) \leq \bigvee \mathcal{T}_{2}\left(B_{i}\right) \leq s .
\end{aligned}
$$

This is a contradiction. Hence $H(\mathcal{T})_{2}\left(\bigvee \eta_{i}\right) \leq \bigvee H(\mathcal{T})_{2}\left(\eta_{i}\right)$ Therefore $(X, H(\mathcal{T}))$ is a MSIFTS.

Next, we show that if $f:(X, \mathcal{T}) \rightarrow(Y, \mathcal{U})$ is SolF continuous, then $f:(X, H(\mathcal{T})) \rightarrow(Y, H(\mathcal{U}))$ is MSIF continuous. Let $H(\mathcal{T})=\left(H(\mathcal{T})_{1}, H(\mathcal{T})_{2}\right), H(\mathcal{U})=\left(H(\mathcal{U})_{1}, H(\mathcal{U})_{2}\right)$, and $\eta \in I^{X}$. Then

$$
\begin{aligned}
H(\mathcal{U})_{1}(\eta) & =\bigvee\left\{\mathcal{U}_{1}(A) \mid \tilde{1}-\gamma_{A}=\eta\right\} \\
& \leq \bigvee\left\{\mathcal{T}_{1}\left(f^{-1}(A)\right) \mid \tilde{1}-\gamma_{f^{-1}(A)}=f^{-1}(\eta)\right\} \\
& \leq \bigvee\left\{\mathcal{T}_{1}(C) \mid \tilde{1}-\gamma_{C}=f^{-1}(\eta)\right\} \\
& =H(\mathcal{T})_{1}\left(f^{-1}(\eta)\right)
\end{aligned}
$$

and

$$
\begin{aligned}
H(\mathcal{U})_{2}(\eta) & =\bigwedge\left\{\mathcal{U}_{2}(A) \mid \tilde{1}-\gamma_{A}=\eta\right\} \\
& \geq \bigwedge\left\{\mathcal{T}_{2}\left(f^{-1}(A)\right) \mid \tilde{1}-\gamma_{f^{-1}(A)}=f^{-1}(\eta)\right\} \\
& \geq \bigwedge\left\{\mathcal{T}_{2}(C) \mid \tilde{1}-\gamma_{C}=f^{-1}(\eta)\right\} \\
& =H(\mathcal{T})_{2}\left(f^{-1}(\eta)\right) .
\end{aligned}
$$

Therefore $H$ is a functor.

Theorem 3.5. Define a functor $K:$ MSIFTop $\rightarrow$ SoIFTop by $K(X, T)=(X, K(T))$ and $K(f)=f$, where $K(T)=$ $\left(K(T)_{1}, K(T)_{2}\right), K(T)_{1}(A)=T_{1}\left(\tilde{1}-\gamma_{A}\right)$, and $K(T)_{2}(A)=$ $T_{2}\left(\tilde{1}-\gamma_{A}\right)$. Then $K$ is a functor.

Proof. First, we show that $K(T)$ is a SoIFT. Clearly,

$$
K(T)_{1}(A)+K(T)_{2}(A)=T_{1}\left(\tilde{1}-\gamma_{A}\right)+T_{2}\left(\tilde{1}-\gamma_{A}\right) \leq 1
$$

for any $A \in I(X)$.

(1) $K(T)_{1}(\underline{0})=T_{1}\left(\tilde{1}-\gamma_{0}\right)=T_{1}(\tilde{0})=1, K(T)_{1}(\underline{1})=$ $T_{1}\left(\tilde{1}-\gamma_{1}\right)=T_{1}(\tilde{1})=1, K(T)_{2}(\underline{0})=T_{2}\left(\tilde{1}-\gamma_{0}\right)=T_{2}(\tilde{0})=$ 0 , and $K(T)_{2}(\underline{1})=T_{2}\left(\tilde{1}-\gamma_{1}\right)=T_{2}(\tilde{1})=0$.

(2) Let $A, B \in I(X)$. Then

$$
\begin{aligned}
K(T)_{1}(A \cap B) & =T_{1}\left(\tilde{1}-\gamma_{A \cap B}\right)=T_{1}\left(\tilde{1}-\gamma_{A} \vee \gamma_{B}\right) \\
& =T_{1}\left(\left(\tilde{1}-\gamma_{A}\right) \wedge\left(\tilde{1}-\gamma_{B}\right)\right) \\
& \geq T_{1}\left(\tilde{1}-\gamma_{A}\right) \wedge T_{1}\left(\tilde{1}-\gamma_{B}\right) \\
& =K(T)_{1}(A) \wedge K(T)_{1}(B)
\end{aligned}
$$

and

$$
\begin{aligned}
K(T)_{2}(A \cap B) & =T_{2}\left(\tilde{1}-\gamma_{A \cap B}\right) \\
& =T_{2}\left(\left(\tilde{1}-\gamma_{A}\right) \wedge\left(\tilde{1}-\gamma_{B}\right)\right) \\
& \leq T_{2}\left(\tilde{1}-\gamma_{A}\right) \vee T_{2}\left(\tilde{1}-\gamma_{B}\right) \\
& =K(T)_{2}(A) \vee K(T)_{2}(B) .
\end{aligned}
$$

(3) Let $A_{i} \in I(X)$ for each $i$. Then

$$
\begin{aligned}
K(T)_{1}\left(\bigcup A_{i}\right) & =T_{1}\left(\tilde{1}-\gamma_{\cup A_{i}}\right)=T_{1}\left(\bigvee\left(\tilde{1}-\gamma_{A_{i}}\right)\right) \\
& \geq \bigwedge T_{1}\left(\tilde{1}-\gamma_{A_{i}}\right)=\bigwedge K(T)_{1}\left(A_{i}\right)
\end{aligned}
$$

and

$$
\begin{aligned}
K(T)_{2}\left(\bigcup A_{i}\right) & =T_{2}\left(\tilde{1}-\gamma_{\cup A_{i}}\right)=T_{2}\left(\bigvee\left(\tilde{1}-\gamma_{A_{i}}\right)\right) \\
& \leq \bigvee T_{2}\left(\tilde{1}-\gamma_{A_{i}}\right)=\bigvee K(T)_{2}\left(A_{i}\right)
\end{aligned}
$$

Thus $(X, K(T))$ is a SoIFTS.

Finally, we show that if $f:(X, T) \rightarrow(Y, U)$ is MSIF continuous, then $f:(X, K(T)) \rightarrow(Y, K(U))$ is SoIF continuous. Let $B=\left(\mu_{B}, \gamma_{B}\right) \in I(Y)$. Then

$$
\begin{aligned}
K(U)_{1}(B) & =U_{1}\left(\tilde{1}-\gamma_{B}\right) \leq T_{1}\left(f^{-1}\left(\tilde{1}-\gamma_{B}\right)\right) \\
& =T_{1}\left(\tilde{1}-\gamma_{f^{-1}(B)}\right)=K(T)_{1}\left(f^{-1}(B)\right)
\end{aligned}
$$


and

$$
\begin{aligned}
K(U)_{2}(B) & =U_{2}\left(\tilde{1}-\gamma_{B}\right) \geq T_{2}\left(f^{-1}\left(\tilde{1}-\gamma_{B}\right)\right) \\
& =T_{2}\left(\tilde{1}-\gamma_{f^{-1}(B)}\right)=K(T)_{2}\left(f^{-1}(B)\right) .
\end{aligned}
$$

Hence $f:(X, K(T)) \rightarrow(Y, K(U))$ is SoIF continuous. Consequently, $K$ is a functor.

Theorem 3.6. The functor $K:$ MSIFTop $\rightarrow$ SoIFTop is a left adjoint of $H$ : SoIFTop $\rightarrow$ MSIFTop.

Proof. For any $(X, T)$ in MSIFTop and $\eta \in I^{X}$,

$$
\begin{aligned}
& H K(T)(\eta) \\
= & \left(\bigvee\left\{K(T)_{1}(A) \mid \tilde{1}-\gamma_{A}=\eta\right\}, \bigwedge\left\{K(T)_{2}(A) \mid \tilde{1}-\gamma_{A}=\eta\right\}\right) \\
= & \left(\bigvee\left\{T_{1}\left(\tilde{1}-\gamma_{A}\right) \mid \tilde{1}-\gamma_{A}=\eta\right\}, \bigwedge\left\{T_{2}\left(\tilde{1}-\gamma_{A}\right) \mid \tilde{1}-\gamma_{A}=\eta\right\}\right) \\
= & \left(T_{1}(\eta), T_{2}(\eta)\right)=T(\eta) .
\end{aligned}
$$

Hence $1_{X}:(X, T) \rightarrow H K(X, T)=(X, T)$ is a MSIF continuous mapping. Consider $(Y, \mathcal{U}) \in$ SoIFTop and a MSIF continuous mapping $f:(X, T) \rightarrow H(Y, \mathcal{U})$. In order to show that $f: K(X, T) \rightarrow(Y, \mathcal{U})$ is a SoIF continuous mapping, let $B \in I(Y)$. Then

$$
\begin{aligned}
K(T)_{1}\left(f^{-1}(B)\right) & =T_{1}\left(\tilde{1}-\gamma_{f^{-1}(B)}\right) \\
& =T_{1}\left(f^{-1}\left(\tilde{1}-\gamma_{B}\right)\right) \\
& \geq H(\mathcal{U})_{1}\left(\tilde{1}-\gamma_{B}\right) \\
& =\bigvee\left\{\mathcal{U}_{1}(C) \mid \tilde{1}-\gamma_{C}=\tilde{1}-\gamma_{B}\right\} \\
& \geq \mathcal{U}_{1}(B)
\end{aligned}
$$

and

$$
\begin{aligned}
K(T)_{2}\left(f^{-1}(B)\right) & =T_{2}\left(\tilde{1}-\gamma_{f^{-1}(B)}\right) \\
& =T_{2}\left(f^{-1}\left(\tilde{1}-\gamma_{B}\right)\right) \\
& \leq H(\mathcal{U})_{2}\left(\tilde{1}-\gamma_{B}\right) \\
& =\bigwedge\left\{\mathcal{U}_{2}(C) \mid \tilde{1}-\gamma_{C}=\tilde{1}-\gamma_{B}\right\} \\
& \leq \mathcal{U}_{2}(B) .
\end{aligned}
$$

Thus $f:(X, K(T)) \rightarrow(Y, \mathcal{U})$ is SoIF continuous. Hence $1_{X}$ is a $K$-universal mapping for $(X, T)$ in MSIFTop.

Let $(r, s)$-gMSIFTop be the category of all $(r, s)$-th graded intuitionistic fuzzy topological spaces in Mondal and Samanta's sense and MSIF continuous mappings, and let CFTop be the category of all Chang's fuzzy topological spaces and fuzzy continuous mappings.

Theorem 3.7. Two categories CFTop and $(r, s)$-gMSIFTop are isomorphic.

Proof. Define $F$ : CFTop $\rightarrow(r, s)$-gMSIFTop by $F(X, T)=$ $(X, F(T))$ and $F(f)=f$, where

$$
F(T)(\eta)=T^{(r, s)}(\eta)= \begin{cases}(1,0) & \text { if } \eta=\tilde{0}, \tilde{1}, \\ (r, s) & \text { if } \eta \in T-\{\tilde{0}, \tilde{1}\} \\ (0,1) & \text { otherwise. }\end{cases}
$$

Define $G:(r, s)$-gMSIFTop $\rightarrow$ CFTop by $G(X, \mathcal{T})=$ $(X, G(\mathcal{T}))$ and $G(f)=f$, where

$$
G(\mathcal{T})=\mathcal{T}_{(r, s)}=\left\{\eta \in I^{X} \mid \mathcal{T}_{1}(\eta) \geq r \text { and } \mathcal{T}_{2}(\eta) \leq s\right\}
$$

Then $F$ and $G$ are functors. Obviously, $G F(T)=G\left(T^{(r, s)}\right)=$ $\left(T^{(r, s)}\right)_{(r, s)}=T$ and $F G(\mathcal{T})=F\left(\mathcal{T}_{(r, s)}\right)=\left(\mathcal{T}_{(r, s)}\right)^{(r, s)}=$ $\mathcal{T}$. Hence CFTop and $(r, s)$-gMSIFTop are isomorphic.

Theorem 3.8. The category $(r, s)$-gMSIFTop is a bireflective full subcategory of MSIFTop.

Proof. Obviously, $(r, s)$-gMSIFTop is a full subcategory of MSIFTop. Let $(X, T)$ be an object of MSIFTop. Then for each $(r, s) \in I \otimes I,\left(X,\left(T_{(r, s)}\right)^{(r, s)}\right)$ is an object of $(r, s)$ gMSIFTop and $1_{X}:(X, T) \rightarrow\left(X,\left(T_{(r, s)}\right)^{(r, s)}\right)$ is a MSIF continuous mapping. Let $(Y, U)$ be an object of the category $(r, s)$-gMSIFTop and $f:(X, T) \rightarrow(Y, U)$ a MSIF continuous mapping. we need only to check that $f:\left(X,\left(T_{(r, s)}\right)^{(r, s)}\right) \rightarrow$ $(Y, U)$ is a MSIF continuous mapping. Since $(Y, U) \in(r, s)$ gMSIFTop, $U(\eta)=(1,0),(r, s)$, or $(0,1)$. Let $U(\eta)=$ $(1,0)$. Then $\eta=\tilde{0}$ or $\tilde{1}$. In fact,

$$
\left(T_{(r, s)}\right)^{(r, s)}\left(f^{-1}(\tilde{0})\right)=\left(T_{(r, s)}\right)^{(r, s)}(\tilde{0})=(1,0)=U(\tilde{0})
$$

and

$$
\left(T_{(r, s)}\right)^{(r, s)}\left(f^{-1}(\tilde{1})\right)=\left(T_{(r, s)}\right)^{(r, s)}(\tilde{1})=(1,0)=U(\tilde{1}) .
$$

In case $U(\eta)=(0,1)$, clearly $U(\eta) \leq\left(T_{(r, s)}\right)^{(r, s)}\left(f^{-1}(\eta)\right)$. Let $U(\eta)=(r, s)$. Since $f:(X, T) \rightarrow(Y, U)$ is MSIF continuous, $T\left(f^{-1}(\eta)\right) \geq U(\eta)=(r, s)$. Thus $f^{-1}(\eta) \in T_{(r, s)}$, and hence $\left(T_{(r, s)}\right)^{(r, s)}\left(f^{-1}(\eta)\right)=(r, s)=U(\eta)$. Therefore $f:\left(X,\left(T_{(r, s)}\right)^{(r, s)}\right) \rightarrow(Y, U)$ is a MSIF continuous mapping.

From the above theorems, we have the follwing main result. 
Theorem 3.9. The category CFTop is a bireflective full subcategory of MSIFTop.

\section{Conclusion}

We obtained two types of adjoint functors between the category of intuitionistic fuzzy topological spaces in Mondal and Samanta's sense, and the category of intuitionistic fuzzy topological spaces in Šostak's sense. Also, we revealed that the category of Chang's fuzzy topological spaces is a bireflective full subcategory of the category of intuitionistic fuzzy topological spaces in Mondal and Samanta's sense.

In further research, we will investigate other properties of the category of intuitionistic fuzzy topological spaces in Šostak's sense.

\section{Acknowledgments}

This work was supported by the research grant of Chungbuk National University in 2013.

\section{References}

[1] K. T. Atanassov, Intuitionistic fuzzy sets, Fuzzy Sets and Systems 20 (1986), 87-96.

[2] C. L. Chang, Fuzzy topological spaces, J. Math. Anal. Appl. 24 (1968), 182-190.

[3] K. C. Chattopadhyay, R. N. Hazra, and S. K. Samanta, Gradation of openness : Fuzzy topology, Fuzzy Sets and Systems 49 (1992), 237-242.

[4] D. Çoker, An introduction to intuitionistic fuzzy topological spaces, Fuzzy Sets and Systems 88 (1997), 81-89.

[5] D. Coker and M. Demirci, An introduction to intuitionistic fuzzy topological spaces in Sostak's sense, BUSEFAL 67 (1996), 67-76.

[6] Seok Jong Lee and Eun Pyo Lee, The category of intuitionistic fuzzy topological spaces, Bull. Korean Math. Soc. 37 (2000), no. 1, 63-76.
[7] Tapas Kumar Mondal and S.K. Samanta, On intuitionistic gradation of openness, Fuzzy Sets and Systems 131 (2002), 323-336.

[8] Sung Wook Park, Eun Pyo Lee, and Hyuk Han, The category of intutionistic fuzzy topological spaces in šostak's sense, Journal of applied mathematics and computing 13 (2003), no. 1-2, 487-500.

[9] A. A. Ramadan, Smooth topological spaces, Fuzzy Sets and Systems 48 (1992), 371-375.

[10] A. P. Šostak, On a fuzzy topological structure, Suppl. Rend. Circ. Matem. Janos Palermo, Sr. II 11 (1985), 89103.

[11] L. A. Zadeh, Fuzzy sets, Information and Control 8 (1965), 338-353.

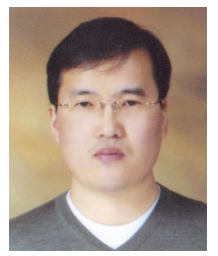

Jin Tae Kim received the Ph. D. degree from Chungbuk National University in 2012. His research interests include general topology and fuzzy topology. He is a member of KIIS and KMS.

E-mail: kjtmath@hanmail.net

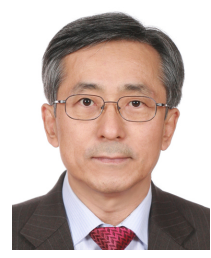

Seok Jong Lee received the M. S. and Ph. D. degrees from Yonsei University in 1986 and 1990, respectively. He is a professor at the Department of Mathematics, Chungbuk National University since 1989 . He was a visiting scholar in Carleton University from 1995 to 1996, and Wayne State University from 2003 to 2004. His research interests include general topology and fuzzy topology. $\mathrm{He}$ is a member of KIIS, KMS, and CMS.

E-mail: sjl@cbnu.ac.kr 